\title{
Oxidative stress preconditioning of mouse perivascular myogenic progenitors selects a subpopulation of cells with a distinct survival advantage in vitro and in vivo
}

\author{
Cesare Gargioli', Giuseppina Turturici², Maria M. Barreca², Walter Spinello², Claudia Fuoco', Stefano Testa',
} Salvatore Feo², Stefano M. Cannata', Giulio Cossu³, Gabriella Sconzo ${ }^{2}$ and Fabiana Geraci $\mathbb{B}^{2,4}$

\begin{abstract}
Cell engraftment, survival and integration during transplantation procedures represent the crux of cell-based therapies. Thus, there have been many studies focused on improving cell viability upon implantation. We used severe oxidative stress to select for a mouse mesoangioblast subpopulation in vitro and found that this subpopulation retained selfrenewal and myogenic differentiation capacities while notably enhancing cell survival, proliferation and migration relative to unselected cells. Additionally, this subpopulation of cells presented different resistance and recovery properties upon oxidative stress treatment, demonstrating select advantages over parental mesoangioblasts in our experimental analysis. Specifically, the cells were resistant to oxidative environments, demonstrating survival, continuous self-renewal and improved migration capability. The primary outcome of the selected cells was determined in in vivo experiments in which immunocompromised dystrophic mice were injected intramuscularly in the tibialis anterior with selected or non-selected mesoangioblasts. Resistant mesoangioblasts exhibited markedly enhanced survival and integration into the host skeletal muscle, accounting for a more than $70 \%$ increase in engraftment compared with that of the unselected mesoangioblast cell population and leading to remarkable muscle recovery. Thus, the positive effects of sorting on mesoangioblast cell behaviour in vitro and in vivo suggest that a selection step involving oxidative stress preconditioning may provide a novel methodology to select for resistant cells for use in regenerative tissue applications to prevent high mortality rates upon transplantation.
\end{abstract}

\section{Introduction}

The release of several types of factors, such as cytokines and growth factors, from damaged tissues stimulates both resident and circulating stem cells to initiate tissue repair programmes. ${ }^{1-3}$ However, the therapeutic efficacy of stem cells is compromised by reduced homing towards the target site $e^{4,5}$ and by the cytotoxic environment, which

\footnotetext{
Correspondence: Fabiana Geraci (fabiana.geraci@unipa.it)

'Department of Biology, University of Rome "Tor Vergata", Rome 00133, Italy

2Department of Biological, Chemical and Pharmaceutical Sciences and

Technologies, University of Palermo, Palermo 90128, Italy

Full list of author information is available at the end of the article

Cesare Gargioli and Giuseppini Turturici contributed equally to this work.

Edited by Y. Wang
}

causes massive cell death during the first several days post-transplantation. ${ }^{5-9}$ For this reason, enhancing in vivo stem cell viability may be a key step in improving the outcomes of cell-based therapies. The microenvironment within damaged tissues is unfavourable for stem cell survival due to hypoxia, inflammatory mediators, a lack of glucose or serum and oxidative stress, with the latter being particularly detrimental. ${ }^{6,10,11}$ In particular, hydrogen peroxide $\left(\mathrm{H}_{2} \mathrm{O}_{2}\right)$, a reactive oxygen species (ROS) that diffuses freely into and out of cells, ${ }^{12,13}$ may play a significant role in inducing the apoptosis or necrosis of injected stem cells. ${ }^{13-15}$ Although the regulation of cell

\section{(c) The Author(s). 2017}

\footnotetext{
(c) (i) Open Access This article is licensed under a Creative Commons Attribution 4.0 International License, which permits use, sharing, adaptation, distribution and reproduction in any medium or format, as long as you give appropriate credit to the original author(s) and the source, provide a link to the Creative Commons license, and indicate if changes were made. The images or other third party material in this article are included in the article's Creative Commons license, unless indicated otherwise in a credit line to the material. If material is not included in the article's Creative Commons license and your intended use is not permitted by statutory regulation or exceeds the permitted use, you will need to obtain permission directly from the copyright holder. To view a copy of this license, visit http://creativecommons.org/licenses/by/4.0/.
} 
death by external oxidative stress has been extensively studied in vitro, these experiments typically use differentiated cells rather than stem cells and focus on events that occur shortly after treatment (i.e., a few minutes later or at most in the first $24 \mathrm{~h}){ }^{16,17}$ In the field of stem cell research, in vitro experiments based on comparative analyses of oxidative stress resistance among mesenchymal stem cells, embryonic stem cells and induced pluripotent stem cells (iPSCs) have shown that iPSCs and embryonic stem cells are less resistant to oxidative stress than mesenchymal stem cells. ${ }^{18}$ However, other studies have demonstrated that oxidative stress induces senescence in human mesenchymal stem cells. ${ }^{19-21}$ Therefore, despite its central role in the development of cell-based therapies, the effects of exogenous oxidative stress on stem cell viability are not well understood. To explore the reasons why only a few stem cells survive after transplantation, we first performed an in vitro study. $\mathrm{H}_{2} \mathrm{O}_{2}$ was used to apply extreme exogenous oxidative stress to mouse mesoangioblast perivascular myogenic progenitors (hereafter referred to as 'mabs' or 'A6 cells') to isolate resistant cells that survived after a long recovery period. The resistant cells (hereafter 'cell clones' or 'H2 cells') exhibited the unusual ability to retain self-renewal capacity in addition to increased migratory and proliferation capabilities with respect to the untreated mab population. Moreover, in vivo experiments involving the intramuscular injection of cell clones into immunocompromised dystrophic mice further highlighted noteworthy improvements in cell survival, migration and engraftment into host skeletal muscle tissue compared with those of unstressed cells. Mabs are easily expandable in vitro and have largely been studied for cell-based therapeutic applications; thus, they are prime candidates for skeletal muscle regeneration and reconstruction. ${ }^{22-27}$ Therefore, mabs derived from the selected subpopulation are better able to tolerate oxidative stress and display distinct survival and integration advantages in vivo upon transplantation, representing an important approach to potentiate improvements in mab-based cell therapy.

\section{Results \\ Different $\mathrm{H}_{2} \mathrm{O}_{2}$ doses and exposure times affect mab cell cycle progression and viability}

To select resistant cells that survive in an oxidative environment, we identified a sub-lethal concentration of $\mathrm{H}_{2} \mathrm{O}_{2}$ that inhibited cell cycle progression and partially killed the treated cells. We analysed the dose responses of

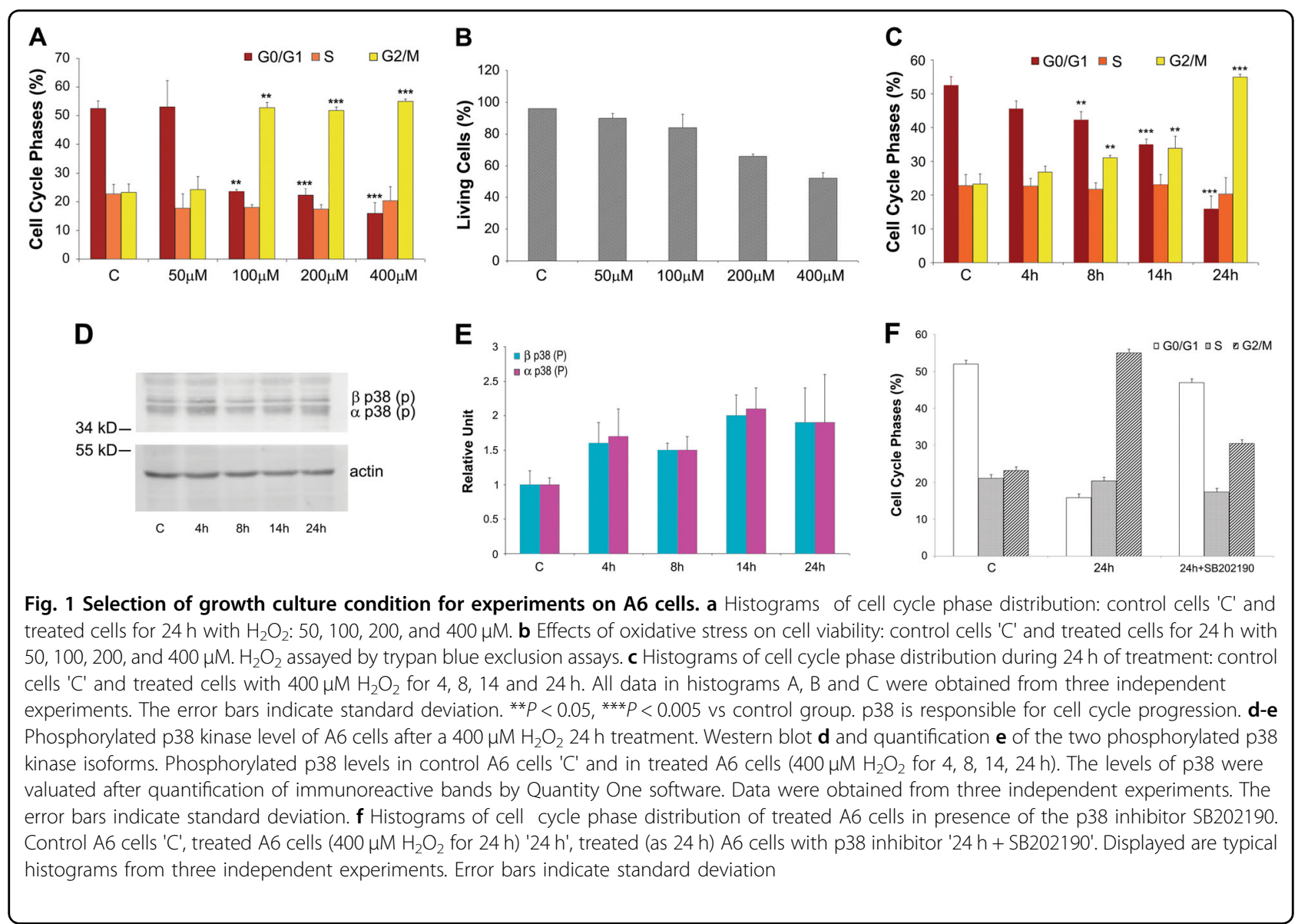


sub-confluent mab cultures treated with varying doses of $\mathrm{H}_{2} \mathrm{O}_{2}$ and determined that treatment with $400 \mu \mathrm{M} \mathrm{H} \mathrm{H}_{2} \mathrm{O}_{2}$ for $24 \mathrm{~h}$ resulted in cell cycle arrest in the $\mathrm{G}_{2} / \mathrm{M}$ phase (Fig. 1a) and 50\% cell survival (Fig. 1b), representing optimal conditions to isolate oxidative stress-resistant mabs. Cell cycle analysis by cytofluorimeter revealed higher $G_{2} / M$ phase arrest after exposure for $24 \mathrm{~h}$ of but not at shorter time points (i.e., 4,8 , or $14 \mathrm{~h}$ ), indicating that arrest was strictly dependent on the length of exposure (Fig. 1c). This high dose was the maximum theoretical $\mathrm{H}_{2} \mathrm{O}_{2}$ concentration achievable in tissues with severe inflammation. Thus, the highest tolerable concentration that blocks mitosis and does not immediately induce complete death in mabs was chosen to select for the oxidative stress-resistant subpopulation.

We also investigated the p38 activation pathway, which may participate in cell cycle arrest during mitosis $\left(G_{2} / M\right.$ phase) due to Cdc25B inhibition resulting from increased phosphorylated p38 MAPK activity. ${ }^{28,29}$ To explore whether this same mechanism occurs in mabs upon oxidative stress exposure $\left(400 \mu \mathrm{M} \mathrm{H}_{2} \mathrm{O}_{2}\right)$, we evaluated $\mathrm{p} 38$ MAPK levels after $\mathrm{H}_{2} \mathrm{O}_{2}$ exposure for different periods of time $(4,8,14$ and $24 \mathrm{~h})$ when we observed mitotic blockade (see Fig. 1c). Western blotting (Figs. 1d, e) showed that both alpha- and beta-phosphorylated isoform levels increased during oxidative stress at these same time points when we observed mitosis blockade. This association appeared functionally relevant because mabs treated with both $400 \mu \mathrm{M} \mathrm{H}_{2} \mathrm{O}_{2}$ and a p38 MAPK inhibitor (SB202190) did not exhibit $\mathrm{G}_{2} / \mathrm{M}$ phase blockade (Fig. 1f). This finding supports a role for p38 MAPK activity in cell cycle blockade due to oxidative stress.

\section{Cell viability, proliferation and ROS generation during the recovery period}

To study long-term survival in $\mathrm{H}_{2} \mathrm{O}_{2}$-treated mabs, specific analyses were performed during the recovery period. Throughout recovery, a portion of the treated cells died, as assessed by trypan blue exclusion assays. This trend began to reverse on the eighth day of recovery (Fig. 2a). Simultaneously, during the 1 days of recovery, the cells were still blocked in $\mathrm{G}_{2} / \mathrm{M}$ phase (Fig. 2b); cell cycle distribution returned to normal on day five. Thus, although the cell cycle was restored, cell death continued daily. The intracellular ROS concentration was maximal on the 1 day of recovery, indicating that at the end of treatment, the stressed cells were producing large quantities of endogenous ROS (Fig. 2c).

\section{$\mathrm{H}_{2} \mathrm{O}_{2}$ mab treatment facilitates the isolation of resistant cell clones}

On day 8, proliferation started to prevail over cell death. Treated cells after day eight of recovery were plated at

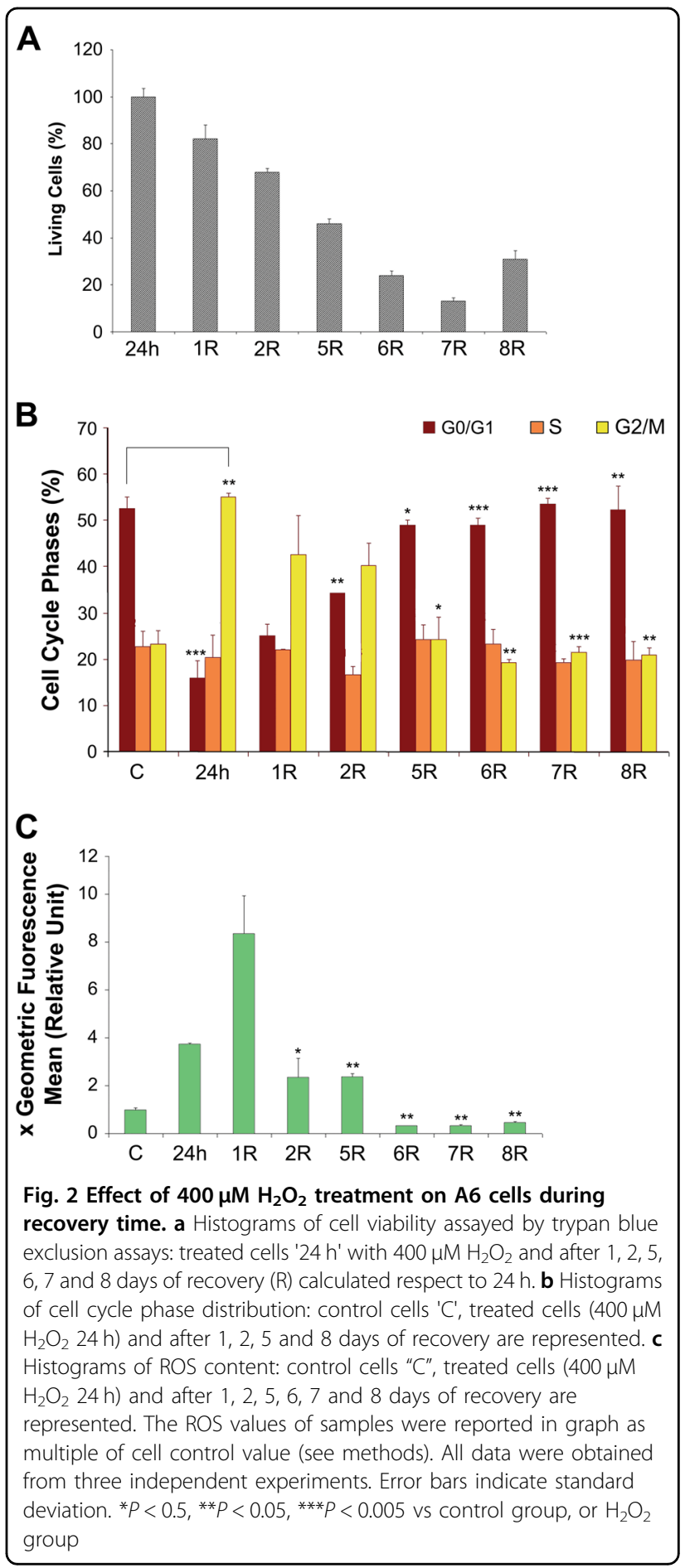

clonal dilutions and subsequently grown for an additional 15 days to sort resistant clones. The plated cells continued to die during the subsequent 15 days of recovery, and only a few cells survived to form clones. The surviving cell clones were then subjected to a second round of oxidative stress by applying the same sub-lethal treatment $(400 \mu \mathrm{M}$ $\mathrm{H}_{2} \mathrm{O}_{2}$ for $24 \mathrm{~h}$ ). Based on a cell cycle analysis of three 


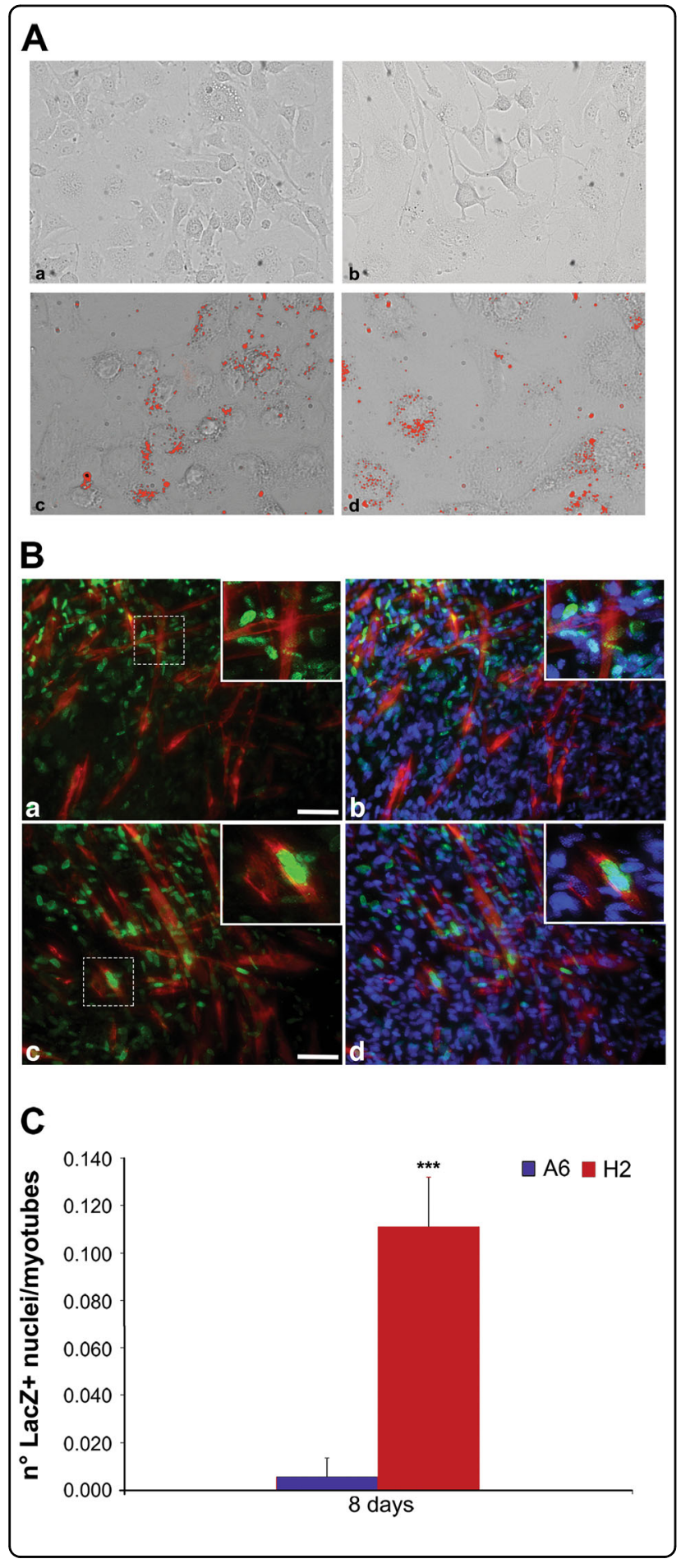

clones (H2, E3, B9), lower percentages of cells were blocked in $G_{2} / M$ phase after treatment than that of mabs (Supplementary Figs. S1A-D). The three cell clones were similar, and for the remainder of the study, we carefully analysed the $\mathrm{H} 2$ clone to determine whether it preserved the mab phenotype while possessing enhanced resistance to oxidative stress.
Fig. 3 Differentiation of $\mathbf{A} 6$ cells and $\mathrm{H} 2$ cell clone in vitro. a A6 cells $\mathbf{a}$ and cell clone $\mathbf{b}$ after differentiation in adipocytes, A6 cells $\mathbf{c}$ and cell clone $\mathbf{d}$ differentiated in adipocyte and stained by oil red. Showed are typical photographs with 20X magnification. Scale bar is $15 \mu \mathrm{m}$. All data were obtained from three independent experiments. $\mathbf{b}$ A6 cells $\mathbf{a}, \mathbf{b}$ and cell clone $\mathbf{c}, \mathbf{d}$ labeled with lentiviral infection with nuclear nLacZ differentiated in myotube in co-culture with $\mathrm{C} 2 \mathrm{C} 12$ mouse myoblast cell line. Immunofluorescence against LacZ (green) and Myosin Heavy Chain (MyHC) (red) revealing $\mathrm{H} 2$ nuclei participation in fusing and forming myotubes $\mathbf{c}$ while A6 nuclei remain undifferentiated outward $\mathrm{MyHC}$ positive myofibers $\mathbf{a}$; nuclei are counterstained with DAPI (blue) $\mathbf{b}$, $\mathbf{d}$. The inserts are enlarged view of the dashed area, highlighting $\mathrm{H} 2$ enrolment into myotube formation. Scale bar values: $\mathbf{a}, \mathbf{c}=25 \mu \mathrm{m}$; inserts $=10 \mu \mathrm{m}$. $\mathbf{c}$ Number of LacZ positive nuclei of A6 cells and cell clone in myotubes. Error bars indicate standard deviation. ${ }^{* *} P<0.005$

\section{The $\mathrm{H} 2$ clone retains the basal mab phenotype}

To determine whether the $\mathrm{H} 2$ cell clone retained the mab phenotype under normal growth conditions, we assessed morphology, cell doubling time, intracellular ROS levels, expression patterns of certain stemness genes, and differentiation capabilities. In general, there were no differences between mabs and the $\mathrm{H} 2$ cell clone among the examined parameters (Supplementary Figs. S2A-C and Fig. 3a); the exception was the myogenic differentiation of the $\mathrm{H} 2$ cell clone, which demonstrated an improved ability to form myotubes in co-culture with the $\mathrm{C} 2 \mathrm{C} 12$ mouse myoblast cell line (Fig. 3b). To evaluate the myogenic capabilities of selected and unselected mabs, lentiviral infection was performed to label the cells with nuclear $\beta$ - galactosidase $(n L a c Z)^{26,30}$, and the effective participation of the mab nuclei in myotube formation was evaluated, revealing a remarkable increase in $\mathrm{H} 2$ myogenic activity compared with that of its A6 counterpart (Fig. 3c).

\section{Oxidative stress does not affect $\mathrm{H} 2$ clone viability, proliferation and migration capacity}

The sorted cell clone exhibited a behavioural alteration in the presence of an oxidative environment. The cell clone and mabs were subjected to severe oxidative stress using $200 \mu \mathrm{M} \mathrm{H}_{2} \mathrm{O}_{2}$ for $24 \mathrm{~h}$; this concentration may be similar to that encountered in vivo in chronically inflamed tissues. According to a cell cycle analysis, this lower $\mathrm{H}_{2} \mathrm{O}_{2}$ dose arrested mabs in $\mathrm{G}_{2} / \mathrm{M}$ phase (Fig. 4a) during oxidative stress and recovery, as did the higher dose $(400 \mu \mathrm{M})$ (see Fig. 2b). In contrast, the cell clone was not arrested in $\mathrm{G}_{2} /$ $M$ phase, resulting in further analyses (Fig. 4b). We next evaluated the effects of $\mathrm{H}_{2} \mathrm{O}_{2}$ on the viability of both A6 and $\mathrm{H} 2$ cells by analysing the cell percentages in different stages of apoptosis using flow cytometry after performing Annexin V-PE/SYTOX Green double staining, which distinguishes between different stages of apoptotic cell death. After $\mathrm{H}_{2} \mathrm{O}_{2}$ treatment, both cell lines showed a marked increase in apoptosis, although the apoptosis levels 


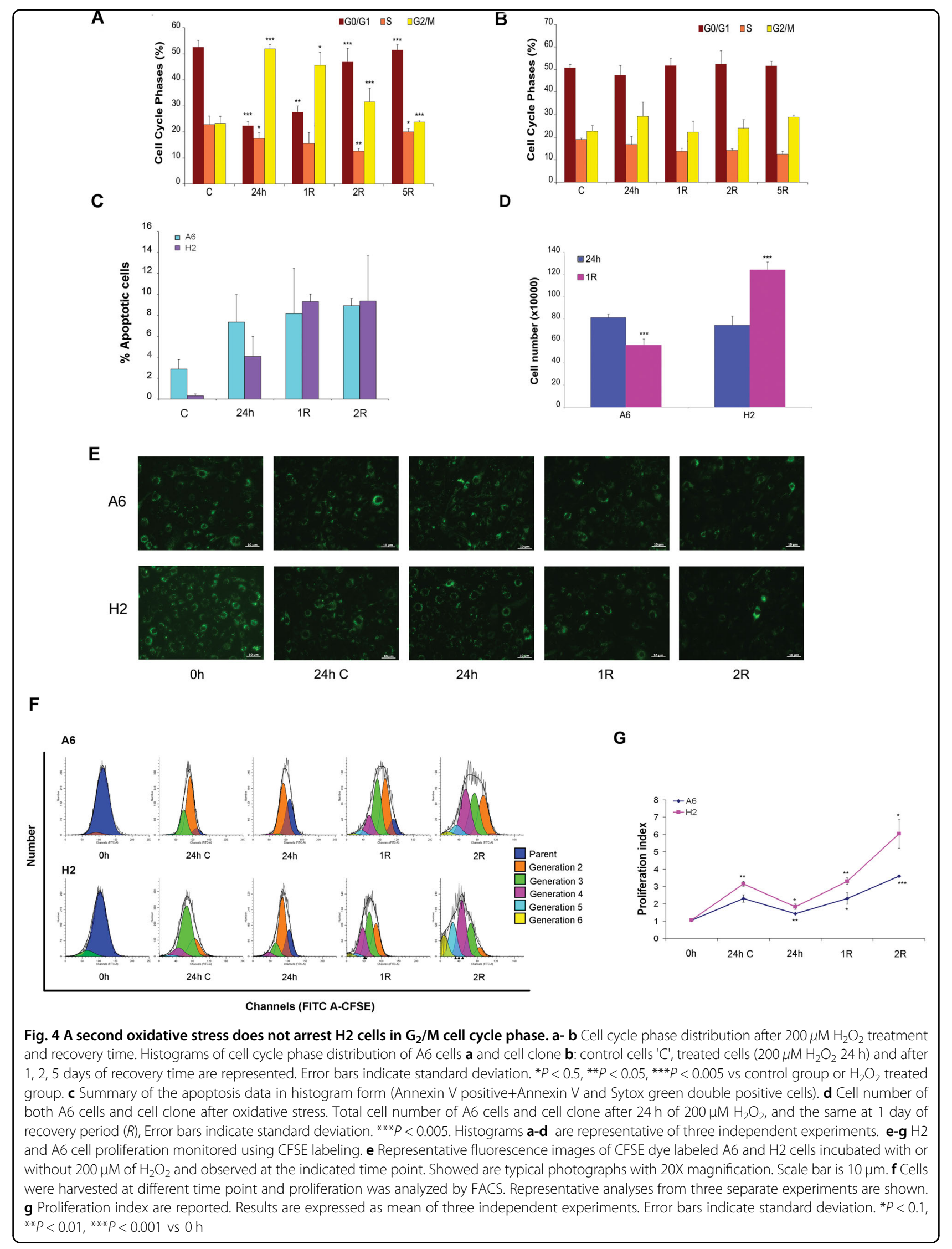




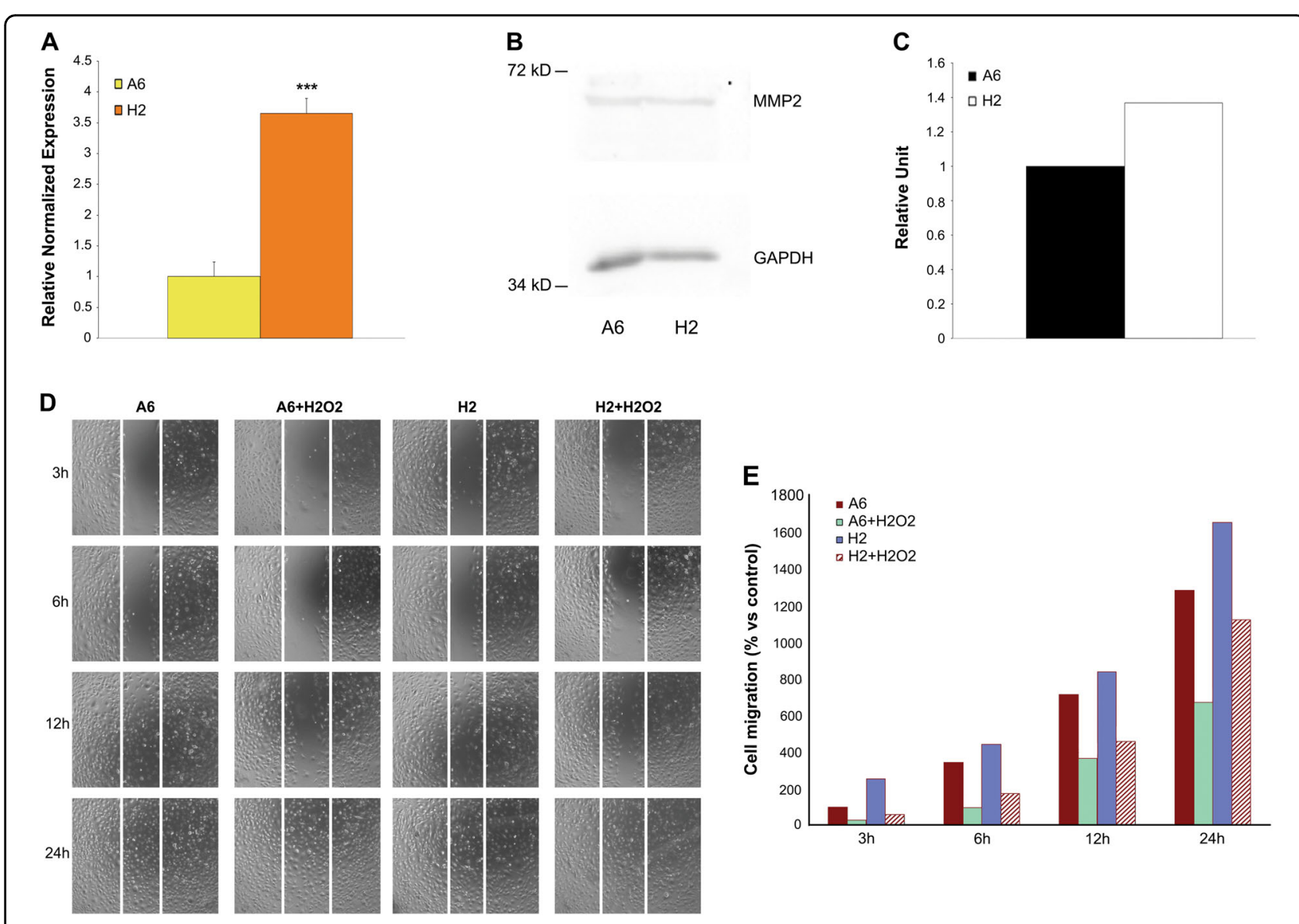

Fig. 5 Different amount of MMP2 in cell clone and A6 cells. a qRT-PCR analysis of MMP2 mRNA in A6 cells and cell clone. Relative quantities of mRNA were first normalized to GAPDH and actin genes, and then A6 samples were arbitrarily set to a value of $1(n=3)$. Data are expressed as mean \pm Standard Error of the Mean. ${ }^{* *} P<0.001$. b, $\mathbf{c}$ Western blot assay and histogram of MMP2 in untreated A6 cells and cell clone. The levels of MMP2 were valuated after quantification of immunoreactive bands, obtained by immunoblot assay, by Quantity one software. d-e Different migration capability of A6 cells vs $\mathrm{H} 2$ cells. Scratch test of A6 cells and cell clone under normal growth conditions and after $200 \mu \mathrm{M} \mathrm{H} \mathrm{H}_{2} \mathrm{O}_{2}$ treatment for $24 \mathrm{~h}$ ' +

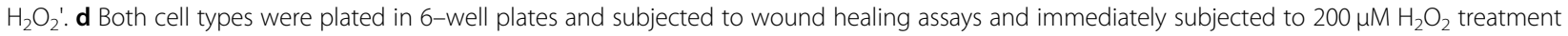
for $24 \mathrm{~h}$. Results are a representative experiment from at least three independently performed experiments with similar results. e The number of migrated cells was recorded and the data of three independent experiments are expressed as means. A6 samples were arbitrarily set to a value of 100

were higher in $\mathrm{A} 6$ than $\mathrm{H} 2$ cells (Fig. 4c), confirming the superior resistance of $\mathrm{H} 2$ cells to oxidative stress. Then, we characterized the growth capacity of this cell clone, which was doubled compared to the total cell number on day one of recovery after stress induction (Fig. 4d). Conversely, the total number of mabs was reduced, suggesting that mabs and the cell clone behaved differently after exposure to oxidative stress. The proliferative responses of both mabs and $\mathrm{H} 2$ cells were also observed after CFSE labelling (Fig. 4e) and quantitatively evaluated using a CFSE-based cytometry assay. After different time points, cell proliferation was assessed by processing the degree of reduction in green fluorescence intensity using ModFit $\mathrm{LT}^{\mathrm{TM}}$ software (Fig. 4f). As shown in Fig. 4g, H2 cells had a higher proliferation index than A6 cells under basal growth conditions. A significant reduction in this index was observed $24 \mathrm{~h}$ after treatment with $200 \mu \mathrm{M} \mathrm{H} \mathrm{H}_{2} \mathrm{O}_{2}$. Despite this reduction, $\mathrm{H} 2$ cells exhibited a proliferation index higher than that of A6 cells one day after $\mathrm{H}_{2} \mathrm{O}_{2}$ removal. Both $\mathrm{H} 2$ and $\mathrm{A} 6$ cells demonstrated proliferative capacities comparable to pre-stressed conditions. The $\mathrm{H} 2$ proliferative index was doubled after 2 days of recovery compared with that of A6 cells.

Another fundamental stem cell function in transplantation applications is migration capacity; therefore, we evaluated the $\mathrm{H} 2$ cell clone for its expression of matrix metalloproteinase 2 (MMP2), which is crucial for migration $^{31}$, to define H2 migratory capacity compared with that of unselected mabs. Real-time PCR and western blot analyses revealed increased MMP2 expression in the H2 cell clone (Fig. 5a-c). To determine whether this increased MMP2 expression was related to enhanced migration 
B
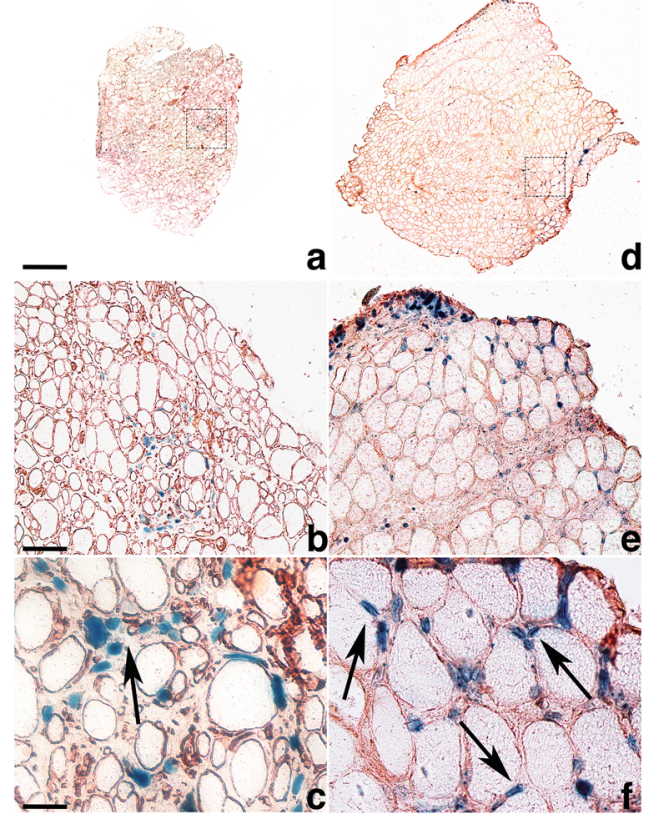

D

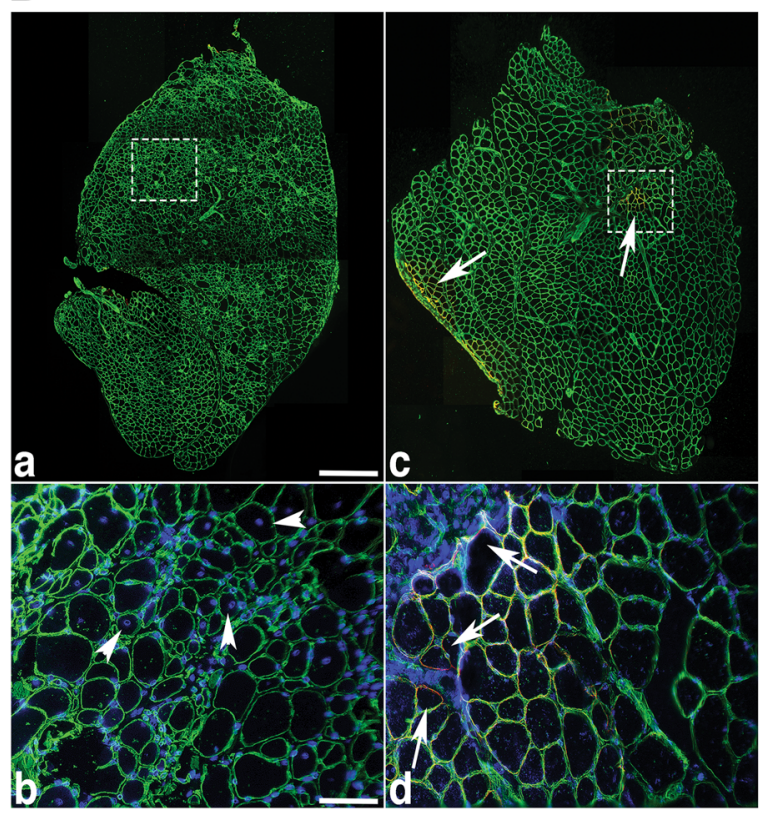

A
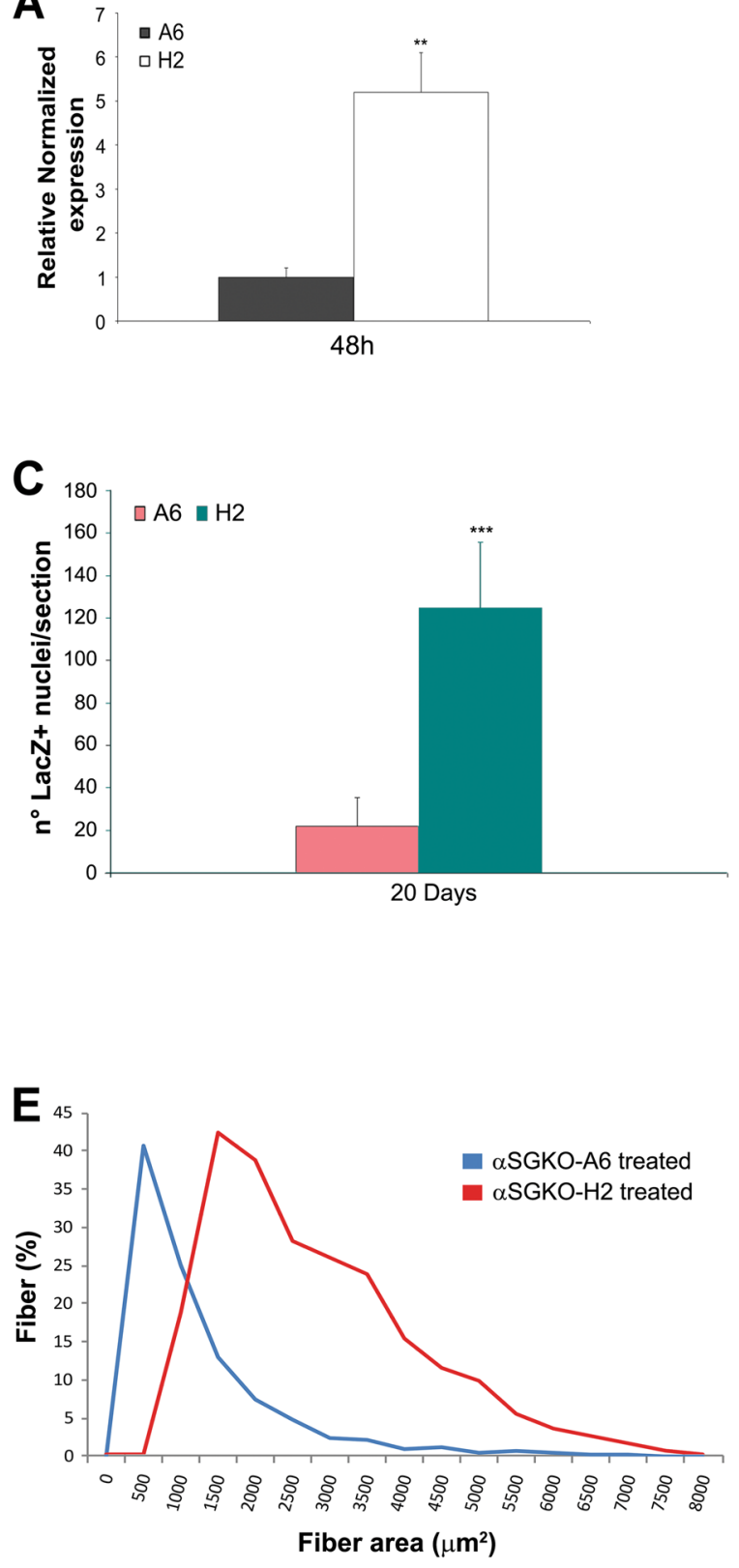

Fig. $6 \mathrm{H} 2$ clone transplanted into mouse immunocompromised dystrophic model is more resistant and have a higher integration capability. a LacZ qRT-PCR of A6 cells and cell clone. RNA was isolated from the treated TA $48 \mathrm{~h}$ after mabs intramuscular injection. b Immunohistochemistry against laminin (brown) and X-Gal staining (blue) on TA section showing A6 cells $\mathbf{a}-\mathbf{c}$ and $\mathrm{H} 2$ cell clone $\mathbf{d}-\mathbf{f}$, modified for nLacZ and implanted intramuscularly in mouse dystrophic model, after 20 days from injection. Dotted boxes in a and d indicate areas of enlarged view (b, e) revealing LacZ positive nuclei into host TA, showing in e integrated LacZ positive nuclei inside regenerating host myofibers. The images in a and $d$ are collage resulting to obtain whole TA section images. a, d Magnification 10X, b, e magnification 20X, c, f magnification 40X. Scale bars: a $300 \mu$ m; b) $40 \mu \mathrm{m} ;$ c $20 \mu \mathrm{m}$. c Number of LacZ positive nuclei of A6 cells and cell clone injected in the muscle. The data are representative from three independently experiments. The error bars indicate standard deviation. ${ }^{*} P<0.05,{ }^{* *} P<0.005$. d Muscle recovery upon 40 days $\mathrm{H} 2$ treatment. a-d Collage resulting images from TA section immunofluorescence against Laminin (green) and aSarcoglycan (aSG) (red) revealing morphology and overall aSG expression recovery areas (arrows in b) upon $\mathrm{H} 2$ intramuscular injection $\mathbf{c}, \mathbf{d}$ comparing with $\mathrm{A} 6$ infusion $\mathbf{a}, \mathbf{b}$. $\mathbf{b}, \mathbf{d}$ Enlarged view of dashed boxes showing still small, centrenucleated degenerating myofibers (arrowheads) after A6 graft $\mathbf{b}$ and $\mathrm{ASG}$ expression (arrows) and recovered morphology - myofibers with uniform size —upon cell clone injection $\mathbf{d}$ nuclei were labeled by DAPI (blue). e Fibres Cross Sectional Analysis (CSA) highlights the muscle recovery due to $\mathrm{H} 2 \mathrm{effect}$ on aSGKO dystrophic muscle, presenting the disappearance of small (under $500 \mu^{2}$ ) degenerating muscle fibres 
capability, we performed a scratch test that revealed effective, increased and rapid cell clone migration under normal growth conditions compared with that of mabs (Fig. 5d, e). We then simulated an in vivo scenario in an in vitro assay and repeated the scratch test in the presence of $200 \mu \mathrm{M} \mathrm{H}_{2} \mathrm{O}_{2}$ (Fig. 5d, e). The $\mathrm{H} 2$ cell clone maintained more rapid migration than treated mabs during the first $24 \mathrm{~h}$ of continuous oxidative stress. Based on these results, the $\mathrm{H} 2$ cell clone subpopulation was more resistant to an oxidative environment.

\section{In vivo transplantation of $\mathrm{H}_{2} \mathrm{O}_{2}$-preconditioned mabs}

To verify the improved survival and migration capacity of the $\mathrm{H} 2$ cell clone, in vivo experiments were performed via the intramuscular injection of cells into an immunocompromised, dystrophic mouse model: SCID/alpha sarcoglycan null mice. ${ }^{32} \mathrm{H} 2$ clone cells and unselected wildtype mabs were modified to express $\mathrm{nLacZ}$, and $5 \times 10^{5}$ cells were implanted into the tibialis anterior (TA) muscles of the same mice. The muscles were then collected for real-time PCR (Fig. 6a) and immunohistochemical analysis (Figs. 6b, c) $48 \mathrm{~h}$ and 20 days, respectively, after injection. RNA was isolated from the treated TAs, and real-time PCR revealed a remarkable increase in LacZ expression related to the numbers of surviving and engrafted mabs upon implantation (Fig. 6a). $\beta$ galactosidase immunostaining also revealed notably high levels of LacZ-positive nuclei in the muscles injected with the $\mathrm{H} 2$ cell clone (Fig. $6 \mathrm{~b} \mathrm{~d}, \mathrm{e}$ ) compared with that in the contralateral TA muscles treated with unselected mabs (Fig. 6b a, b, Supplementary Fig. S3). Moreover, high magnification revealed greater spreading within the injected TAs and remarkable integration of $\mathrm{H} 2$ cell clone nuclei into regenerating myofibres labelled by laminin immunostaining, reflecting peripheral LacZ nuclei inside host muscle fibres (arrows in Supplementary Figs. S3 and arrows in Figs. 6b, f). We speculate that the higher numbers of lacZ-positive cells observed when $\mathrm{H} 2$ cell clones were injected into the TA primarily reflects cell survival; unsorted mabs injected into the dystrophic environment were less resistant and had a lower survival rate (Fig. 6c). Moreover, mice treated with intramuscular H2 cells exhibited a notable ameliorated muscle morphology characterized by more uniform myofibre size and the absence of inflammatory infiltration and degenerating-regenerating areas marked by small fibres, which are clear signs of dystrophy pathology (Fig. 6d). Further supporting this morphofunctional muscle recovery, cross sectional area (CSA) analysis of myofibres clearly revealed the amelioration of muscle fibres upon $\mathrm{H} 2$ cell injection, presenting uniform dispersion of fibre size within the recovered muscle; this was also reflected in the recovery of alpha sarcoglycan expression in mice treated with $\mathrm{H} 2$ cells (Fig. 6e).

\section{Discussion}

The current study reports that a mouse mesoangioblast stem cell line, A6, is a heterogeneous population. This finding, together with the ability to select clones exhibiting particular behaviours after severe oxidative stress, may have important implications. The results obtained in vitro and in vivo suggest that the use of selected cells demonstrating improvements in specific activities is preferable to using the entire population when addressing issues related to tissue repair. Intra-heterogeneity is an important aspect of stem cell biology, as reflected in human mesenchymal stem cells, which are highly complex at the biochemical level. ${ }^{33}$ In some cases, certain clones derived from a stem cell population have more abundant transcripts than the parental population, and vice versa. Clonal and microarray analyses have shown that cardiac progenitors expressing Mesp1 are distinct populations, most likely due to their regional segregation during early gastrulation. ${ }^{34}$ Here, we isolated a subpopulation from mesoangioblast vessel-associated progenitor cells derived from the mouse embryonic dorsal aorta at stage E9.5..$^{22,25,35}$ The selected subpopulation demonstrated excellent survival following severe oxidative stress for $24 \mathrm{~h}$, while cell death occurred for the majority of treated mabs. Published in vitro results for human mesenchymal stem cells have revealed premature cell senescence upon $\mathrm{H}_{2} \mathrm{O}_{2}$ treatment ${ }^{19-21}$ and permanent growth blockage. ${ }^{36}$ Pomduck and colleagues ${ }^{37}$ improved mesenchymal stem cell resistance to oxidative stress by generating cells with specific protein overexpression profiles. Here, we proposed a selection step to isolate oxidative stress-resistant cells from a heterogeneous population and demonstrated its major advantages. Our results provide reliable evidence supporting the idea that selected cells-or the $\mathrm{H} 2$ cell clone-resist cell cycle arrest and are more proliferative. Moreover, the $\mathrm{H} 2$ clone migrates faster than mabs in scratch assays both under physiological growth conditions and oxidative stress, based on the observed increased MMP2 expression in these cells, and possess a greater capacity to differentiate. More importantly, H2 cells transplanted into a mouse model of muscular dystrophy were able to considerably ameliorate muscle morphology and restore alpha sarcoglycan expression.

One lingering question is whether surviving cells became resistant due to the negative effects of oxidative stress or due to alterations that occur prior to stress. A study of mesenchymal stem cells demonstrated that specific $\mathrm{H}_{2} \mathrm{O}_{2}$ concentrations induce cell differentiation via the upregulation of Notch signalling, which promotes cell differentiation upon oxidative induction. ${ }^{38}$ In our case, oxidative stress triggered this change/resistance in only a few cells, specifically a subpopulation of mabs, indicating the heterogeneous nature of the starting mab population. Thus, it is possible to promote the cell survival, migration 
and differentiation capacity of a subpopulation of cells by preconditioning stem cells with an oxidative stress signal, subsequently leaving unaltered or even ameliorating the original capabilities of the preconditioned cells.

In conclusion, our findings highlight the potential of stem cell clone isolation as a therapeutic modality for use in regenerative medicine.

\section{Materials and methods}

\section{Cell culture and treatment}

Mabs were grown on type I collagen treated plates in DMEM (Life Technologies, Carlsbad, CA, USA) supplemented with $10 \%$ fetal bovine serum and $1 \%$ antibiotic and antimycotic (Life Technologies) in a humidified 5\% $\mathrm{CO}_{2}$ atmosphere at $37^{\circ} \mathrm{C}$. To induce oxidative stress, cells were grown in medium with $\mathrm{H}_{2} \mathrm{O}_{2}$ (Sigma, St. Louis, MO, USA) at different concentrations. Mabs were treated with $\mathrm{H}_{2} \mathrm{O}_{2}$ only after reaching the confluence of $80 \%$. We used SB 202190 (10 $\mu \mathrm{M}$ for $30 \mathrm{~min}$ ) (Sigma) as p38 inhibitor.

\section{Trypan blue dye exclusion}

To measure viability, cells were stained with trypan blue (Sigma) dye and then counted as described elsewhere. ${ }^{39}$

\section{Cytofluorimetric analysis}

For cell cycle analysis cells were collected and washed with phosphate buffered saline (PBS) (Life Technologies). Pellets were treated with $0.1 \% \mathrm{C}_{6} \mathrm{H}_{5} \mathrm{Na}_{3} \mathrm{O}_{7}, 0.1 \% \mathrm{NP}-40$, $50 \mu \mathrm{g} / \mathrm{ml}$ propidium iodide (Life Technologies) and 0.06 $\mathrm{mg} / \mathrm{ml}$ RNase A (Sigma) for $45 \mathrm{~min}$ at RT in the dark. The cells were acquired on a BD FACSCanto (BD Biosciences, Franklin Lakes, NJ, USA) using FACSDiva Software. The analysis was performed using Flowing software 1.6.0.

To determine the intracellular ROS content, cells were collected, washed with PBS and incubated with $2^{\prime}, 7^{\prime}$ dichlorfluorescein-diacetate (Sigma) $5 \mu \mathrm{M}$ for $30 \mathrm{~min}$ at $37^{\circ} \mathrm{C}$. The pellet that had been previously washed with PBS was resuspended again in PBS. Cells were acquires on a BD FACSCanto and analyzed using Flowing software 1.6.0.

\section{Preparation of protein extracts}

Protein extracts were prepared as previously described. ${ }^{31}$ Briefly PBS-washed cells were resuspended in lysis buffer (20 mM Hepes, pH 7.9; 0.4 M NaCl; 0.2 mM EDTA; $10 \%$ glycerol; $0.5 \mathrm{mM}$ dithiothreitol; $0.5 \mathrm{mM}$ PMSF; $1 \mathrm{X}$ protease/phosphatase inhibitors, Sigma) and lysed by three cycles of freezing (liquid nitrogen) and thawing $\left(37^{\circ} \mathrm{C}\right.$ water bath). Cell debris was removed by centrifugation at $17,000 \mathrm{rpm}$ for $30 \mathrm{~min}$ at $4{ }^{\circ} \mathrm{C}$.

The extract protein concentrations were determined by the Bradford microassay method (Sigma) using bovine serum albumin as a standard.

\section{Western blot (immunoblot) analysis}

Whole-cell protein extracts were subjected to a $10 \%$ SDS-PAGE and transferred to an ECL-Hybond membrane (GE Healthcare Life Sciences, Little Chalfont, UK) using a mini-electroblot (Biorad, Hercules, CA, USA). After blocking for $2 \mathrm{~h}$ in $5 \%$ nonfat dry milk in TBST buffer $(10 \mathrm{mM}$ Tris- $\mathrm{HCl}, \mathrm{pH} 7.5,150 \mathrm{mM} \mathrm{NaCl}, 0.2 \%$ Tween 20) the membranes were incubated overnight with mouse polyclonal anti-actin (1:1500 dilution, Santa Cruz, Dallas, TX, USA), anti-GAPDH (1:500 dilution Santa Cruz), mouse monoclonal anti-p-p38 (1:200 dilution, Santa Cruz), anti MMP2 (1:1000 dilution, Genetex, Irvine, CA, USA). Peroxidase conjugated anti-rabbit, anti-mouse and anti-rat (1:5000 dilution; Promega, Fitchburg, WI, USA) were used as secondary antibodies. Images are acquired with the Versa-Doc Imaging System (Bio-Rad) and analyzed with the Quantity One software (Biorad).

\section{Adipocyte differentiation assay}

Cells were treated with $10 \mathrm{ng} / \mathrm{ml}$ of dexamethasone (Sigma) in complete medium for 5 days and then analyzed for adipocyte morphology. Cells were washed with PBS, and after were fixed with formaldehyde $10 \%$ (Sigma) for $90 \mathrm{~min}$. Cells were washed three times with PBS and were then incubated with oil red O (Sigma) for $30 \mathrm{~min}$. After cells are washed with PBS and observed.

\section{Scratch (wound) assays}

Scratch assays was performed as previously described. ${ }^{31}$ Confluent monolayer of mabs and cell clone was wounded by using a $200 \mu \mathrm{l}$ pipette tip. Cells were washed with DMEM (Life Technologies) (without serum and antibiotics), to remove any detached cells and photographs of the wound were taken. Treatment with $\mathrm{H}_{2} \mathrm{O}_{2} 200 \mu \mathrm{M}$ (Sigma) was added after wound creation. After 3, 6, 12 and $24 \mathrm{~h}$ the cells were photographed. All images were made at RT in the medium in which the cells were grown, using a contrast phase microscope (Leica DMIL LED, Wetzlar, DE) with a $4 \mathrm{X}$ (HIPLAN NA. 0.10, Leica) objective, magnification 40X and a camera Cool Snap ProColor (Media Cybernetics, Rockville, MD, USA). Four representative extents of cell migration near the wound areas were photographed.

\section{Quantitaive RT-PCR (qRT-PCR)}

Total RNA was extracts with an RNeasy Mini Kit (Qiagen, Hiden, DE), according to the manufacturer's instructions. qRT-PCR was performed on MMP2. GAPDH and actin were used as internal controls. All reactions were performed by using the i-Script One Step RT-PCR Sybr Green Kit (BioRad) according to the manufacturer's instructions. The real time RT-PCR primers are summarized in Table 1. qRT-PCR for MMP2 was performed in a total of $25 \mu$ from a mixture containing 
$0.5 \mu \mathrm{l}$ of Taq Polymerase with Sybr green mix, $12.5 \mu \mathrm{l}$ of $2 \mathrm{X}$ Buffer, $4.5 \mu \mathrm{l}$ of forward primer $(900 \mathrm{nM}), 4.5 \mu \mathrm{l}$ of reverse primer $(900 \mathrm{nM}), 1000 \mathrm{ng}$ of RNA for MMP2 gene. Reactions were run in BioRad CFX-96 detector system under the following conditions: 40 cycles of $95^{\circ} \mathrm{C}$ for $10 \mathrm{~s}, 60^{\circ} \mathrm{C}$ for $30 \mathrm{~s}$, after pre-incubation at $95^{\circ} \mathrm{C}$ for $5 \mathrm{~s}$. All reactions were performed in triplicate. The specificity of the amplification reactions was confirmed by meltingcurve analysis. The fold changes in the mRNA expression level of MMP2 in the two cell lines and control sample were compared using the DDCq method (BioRad CFX Manager Software).

Real time RT-PCR for in vivo mabs injection was performed as described. ${ }^{40}$ Briefly, total RNA was extracted by liquid nitrogen homogenization with TRIzol reagent (Life Technologies) following the manual instruction. The obtained RNA was cleaned and DNase treated with RNeasy Mini Kit (Quiagen). Single-stranded cDNA was synthesized with SuperScript First-Strand RT-PCR system (Life Technologies), according to the protocol supplied by the manufacturer. Real time PCR was performed using SYBR GREEN PCR Master Mix (PE Applied Biosystems Foster City, CA, USA) according to the supplied method by intron-spanning primers:

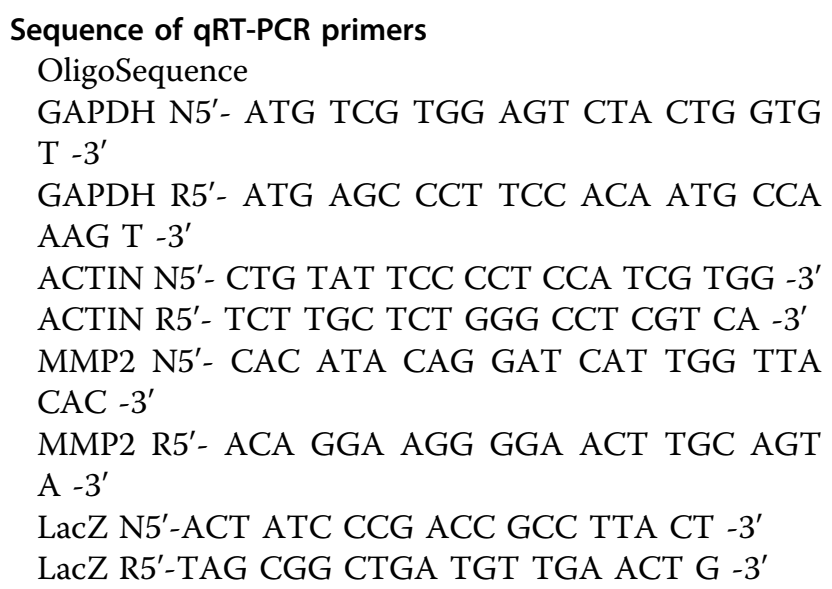

\section{Flow cytometry analysis of cell apoptosis}

Apoptosis was measured with Annexin V/PE apoptosis detection kit (BioVison, San Francisco, CA, USA) according to manufacturer's instructions. Briefly, cells were treated with $200 \mu \mathrm{M} \mathrm{H}_{2} \mathrm{O}_{2}$ for $24 \mathrm{~h}$, harvested and resuspended in binding buffer with the addition of Annexin V-PE and sytox green. Cells were collected and analyzed by cytometry.

\section{Proliferation assay}

A6 and $\mathrm{H} 2$ cells were labeled with CFSE dye using the CFSE Cell Division Tracker Kit (Biolegend, San Diego, CA, USA) according to manufacturer's instructions. This nontoxic cell-permeant fluorescein dye attaches to cytoplasmic components of cells, resulting in uniform brightness. On cell division, the dye is distributed equally (and thus diluted) between daughter cells. Treatment with $\mathrm{H}_{2} \mathrm{O}_{2} 200 \mu \mathrm{M}$ (Sigma) was added after CFSE labeled and at different time point cells were photographed. All images were obtained using a Zeiss Observer D1 inverted microscope (Zeiss, Oberkochen, Germany) equipped with a Zeiss AxioCam MRm and Axiovision Software (Zeiss). Magnification 20X. For FACS analysis cells were harvested at different time point and analyzed by $\mathrm{BD}$ FACSCanto. FITC fluorescence intensities were further analyzed mathematically by ModFit LT $^{\mathrm{TM}} 3.3$ software (Verity Software House Inc., Topsham, ME) to calculate the proliferation index.

\section{Mesoangioblast trasnsduction with lentiviral vectors}

Third-generation lentiviral vectors encoding nuclear $\beta$ Galactosidase were employed for mabs transduction, mabs expressing nuclear LacZ (Mabs-nLacZ) were cultured and analyzed in vitro by myogenic fusion and differentiation, and in vivo by intramuscular injection. ${ }^{26,30}$

\section{Animals and treatments}

Three-months-old SCID (Severe Combined Immune Deficiency) Alpha Sarcoglycan ( $\alpha$-SG) null mice were used for intramuscular injection. ${ }^{31}$ Briefly mice were anesthetized with an intramuscular injection of physiologic saline buffer $(10 \mathrm{ml} / \mathrm{kg})$ containing ketamine $(5 \mathrm{mg} /$ $\mathrm{ml}$ ) (Bayer, Leverkusen, DE) and xylazine $(1 \mathrm{mg} / \mathrm{ml})$ (Bayer). For intramuscular cell delivery, approximately $5 \times 10^{5}$ Mabs-nLacZ were injected into the TA via a $0.20 \mathrm{~mm}$ diameter needle inserted along the cranio-caudal axis TA. Mice were sacrificed at different time points for molecular and morphological analysis.

\section{Immunohistochemistry}

The tissue samples were embedded in O.C.T. (BioOptica, Milano, IT) and flash-frozen in liquid- $\mathrm{N}_{2}$ for $15 \mathrm{sec}$. Sections were cut at a thickness of $8 \mu \mathrm{M}$ using a Leika cryostat. The obtained sections were hydrated with PBS and stained with X-Gal (Thermofisher, Waltham, MA, USA) to reveal $\beta$-galactosidase positive cells as described; ${ }^{26,30}$ briefly sections were washed with PBS (two wash, 5 min each) and incubated $24 \mathrm{~h}$ at $37^{\circ} \mathrm{C}$ with 'X-Gal working solution'. XGal working solution is composed of 'X-gal stock solution' (X-Gal $40 \mathrm{mg} / \mathrm{ml}$ in $\mathrm{N}, \mathrm{N}$-dimethyl formamide stored at $-20^{\circ} \mathrm{C}$ and light protected) diluted $40 \mathrm{X}$ in the 'X-Gal dilution buffer' (Potassium Ferricyanide Crystalline $5 \mathrm{mM}$, Potassium Ferricyanide Trihydrate $5 \mathrm{mM}$, Magnesium Chloride $2 \mathrm{mM}$ in PBS, light protected and stored at $4{ }^{\circ} \mathrm{C}$ ). The sections were then incubated with primary antibody (Rabbit anti-Laminin from SIGMA at a 1:100 final concentration) diluted with blocking buffer (PBS with $0.2 \%$ Triton X-100 and 20\% heat-inactivated goat serum) for 
$20 \mathrm{~min}$ at room temperature (RT). Sections were washed with washing solution (PBS with $0.2 \%$ Triton X-100 and $1 \%$ Bovine Serum Albumine) and then incubated with secondary antibody (HRP-conjugated goat anti-rabbit from Chemicon, diluted 1:500 in 20\% goat serum). The immune reaction was developed using 3-amino-9 ethylcarbazole substrate (AEC, Sigma). Or for H\&E staining, sections were washed with PBS (two wash, 5-10 min each), counterstained with aqueous Eosin (Sigma) and then covered directly with aqueous mounting medium, Aqua Poly/ Mount (Polysciences, Inc., Warrington, PA, USA) The LacZ positive nuclei were counted for three different nonadjacent transverse sections from the largest TA portion for 3 mice per experimental group.

\section{Immunofluorescence}

Immunofluorescence was performed according with previous publication ${ }^{41}$. Briefly, the cell cultures were fixed in $2 \%$ paraformaldehyde (Sigma) for $15 \mathrm{~min}$ at $4{ }^{\circ} \mathrm{C}$, then washed with wash buffer (PBS with $0.2 \%$ Triton X-100) and incubated with primary antibodies diluted with blocking buffer (PBS with 0.2\% Triton X-100 and 20\% heat-inactivated goat serum) for $1 \mathrm{hr}$ at RT. The primary antibodies used for cell culture staining were mouse anti Myosin Heavy Chain (MF20) (Developmental Studies Hybridoma Bank, Iowa city, IA) at 1:2 dilution and rabbit anti-lacZ (Cappel) diluted 1:100. For histological section, collected TA were treated as described previously for sectioning then sections were rehydrated in PBS and soaked in blocking buffer (PBS with $0.2 \%$ Triton X-100 and $20 \%$ heat-inactivated goat serum) for $20 \mathrm{~min}$ at RT. Afterwards the sections washed with washing buffer and incubated with primary antibodies diluted with blocking buffer. the primary antibody used were: rabbit antiLaminin from SIGMA at a 1:100 final concentration and mouse anti- $\alpha$ Sarcglycan (Vector, Ad1/20A6) diluted at 1:100. After several washes with washing buffer, sections were incubated with secondary antibodies diluted with blocking buffer for $1 \mathrm{hr}$ at RT. The secondary antibodies used at 1:500 were goat anti-mouse FITC (Chemicon, Waltham, MA, USA) and goat anti-rabbit Alexa488 (Molecular Probes). Specimens were counterstained with DAPI (1:500, Molecular Probes) 10 minutes at RT to detect nuclei, washed several times with wash buffer and photographed with a Nikon Eclipse TE2000 microscope equipped with a CoolSNAP-MYO CCD camera (Photometrix, Kew, AUS) and MetaMorph software (Molecular Devices, Sunnyvale, CA, USA).

\section{Cross-sectional area analysis}

Cryosections stained with anti-laminin antibody were analyzed using an Image macro which automatically detects single fibres within the laminin fluorescent signal. Wrongly detected fibres were manually corrected before to start the CSA measurement. For each sample, the area of more than two thousand single fibres was measured.

\section{Animal procedures}

Experiments on animal were conducted according to the rules of good animal experimentation I.A.C.U.C. $\mathrm{n}^{\circ}$ 432 of 12 March 2006 and under ethical approval released on 16/09/2011 from Italian Ministry of Health, protocol \#228/2015-PR.

\section{Statistical analysis}

Unpaired two-tailed student's $t$-test for comparison of two independent groups were performed with GraphPad software (GraphPad Software Inc., La Jolla, CA, USA). Quantitative data are represented as the mean \pm standard deviation or SEM of at least three independent experiments.

\section{Acknowledgements}

This research was supported by grants from the University of Palermo and PhD funding.

\section{Author details}

'Department of Biology, University of Rome "Tor Vergata", Rome 00133, Italy. ${ }^{2}$ Department of Biological, Chemical and Pharmaceutical Sciences and Technologies, University of Palermo, Palermo 90128, Italy. ${ }^{3}$ Division of Cell Matrix Biology and Regenerative Medicine, University of Manchester, Oxford Road, M13 9PL Manchester, UK. ${ }^{4}$ Euro-Mediterranean Institute of Science and Technology, Palermo, Italy

\section{Competing interests}

The authors declare that they have no competing financial interests.

\section{Publisher's note}

Springer Nature remains neutral with regard to jurisdictional claims in published maps and institutional affiliations.

\section{Supplementary information}

The online version of this article (https://doi.org/10.1038/s41419-017-0012-9) contains supplementary material.

Received: 2 February 2017 Revised: 7 August 2017 Accepted: 18 September 2017

Published online: 03 January 2018

\section{References}

1. Chavakis, E., Urbich, C. \& Dimmeler, S. Homing and engraftment of progenitor cells: a prerequisite for cell therapy. J. Mol. Cell. Cardiol. 45, 514-522 (2008).

2. Sampaolesi, M. et al. Cell therapy of alpha-sarcoglycan null dystrophic mice through intra-arterial delivery of mesoangioblasts. Science 301, 487-492 (2003).

3. Li, F. et al. Apoptotic cells activate the "phoenix rising" pathway to promote wound healing and tissue regeneration. Sci. Signal. 3, ra13-ra32 (2010).

4. Giannotta, M. et al. Targeting endothelial junctional adhesion molecole-A EPAC/Pap-1 axis as a novel strategy to increase stem cell engraftment in dystrophic muscles. EMBO Mol Med 6, 239-258 (2014).

5. Kang, S. K., Shin, I. S., Ko, M. S., Jo, J. Y. \& Ra, J. C. Journey of mesenchymal stem cells for homing: strategies to enhance efficacy and safety of stem cell therapy. Stem Cells Int 2012, 342968-342978 (2012). 
6. Toma, C., Pittenger, M. F., Cahill, K. S., Byrne, B. J. \& Klesser, P. D. Human mesenchymal stem cells differentiate to cardiomyocyte phenotype in the adult murine heart. Circulation. 105, 93-98 (2002).

7. Mangi, A. A. et al. Mesenchymal stem cells modified with Akt prevent remodeling and restore performance of infarcted hearts. Nat. Med. 9, 1195-1201 (2003).

8. Wei, L. et al. Transplantation of embryonic stem cells overexpressing BCl-2 promotes functional recovery after transient cerebral ischemia. Neurobiol. Dis. 19, 183-193 (2005).

9. Sciorati, $C$. et al. Ex vivo treatment with nitric oxide increases mesoangioblast therapeutic efficacy in muscular dystrophy. J. Cell. Sci. 119, 5114-5123 (2006).

10. Lunde, K. et al. Intracoronary injection of mononuclear bone marrow cells in acute myocardial infarction. N. Engl. J. Med. 355, 1199-1209 (2006).

11. Zhu, W., Chen, X., Hu, S. \& Chen, X. Hypoxia and serum deprivation-induced apoptosis in mesenchymal stem cells. Stem cells 24, 416-425 (2006).

12. Seaver, L. C. \& Imlay, J. A. Hydrogen peroxide fluxes and compartmentalization inside growing Escherichia coli. J. Bacteriol. 183, 7182-7189 (2001).

13. Barbouti, A., Doulias, P. T., Nousis, L., Tenopoulou, M. \& Galaris, D. DNA damage and apoptosis in hydrogen peroxide-exposed Jurkat cells: bolus addition versus continuous generation of $\mathrm{H}_{2} \mathrm{O}_{2}$. Free. Radic. Biol. Med. 33, 691-702 (2002).

14. Chandra, J., Samali, A. \& Orrenius, S. Triggering and modulation of apoptosis by oxidative stress. Free. Radic. Biol. Med. 29, 323-333 (2000).

15. Antunes, F. \& Cadenas, E. Cellular titration of apoptosis with steady state concentrations of $\mathrm{H}_{2} \mathrm{O}_{2}$ : submicromolar levels of $\mathrm{H}_{2} \mathrm{O}_{2}$ induces apoptosis through Fenton chemistry independent of the cellular thiol state. Free Radic Biol Med 30, 1008-1018 (2001).

16. Mo, J. S. et al. Notch1 modulates oxidative stress induced cell death through suppression of apoptosis signal-regulating kinase 1. Proc Natl Acad Sci U S A 110, 6865-6870 (2013).

17. Day, R. M. \& Suzuki, Y. J. Cell proliferation, reactive oxygen and cellular glutathione. Dose Response 3, 425-442 (2006).

18. Asensi, K. D. et al. Reprogramming to a pluripotent state modifies mesenchymal stem cell resistance to oxidative stress. J. Cell. Mol. Med. 18, 824-831 (2014).

19. Brandl, A., Meyer, M., Bechmann, V., Nerlich, M. \& Angele, P. Oxidative stress induces senescence in human mesenchymal stem cells. Exp. Cell. Res. $\mathbf{3 1 7}$ 1541-1547 (2011).

20. Kim, J. S. et al. Proteomic and metabolomic analysis of $\mathrm{H}_{2} \mathrm{O}_{2}$-induced premature senescent human mesenchymal stem cells. Exp. Gerontol. 46, 500-510 (2011).

21. Ko, E., Lee, K. Y. \& Hwang, D. S. Human umbilical cord blood-derived mesenchymal stem cells undergo cellular senescence in response to oxidative stress. Stem. Cells. Dev. 21, 1877-1886 (2012).

22. Minasi, M. G. et al. The meso-angioblast: a multipotent, self-renewing cell that originates from the dorsal aorta and differentiates into most mesodermal tissues. Development 129, 2773-2783 (2002).

23. Sampaolesi, M. et al. Mesoangioblast stem cells ameliorate muscle function in dystrophic dogs. Nature. 444, 574-579 (2006).

24. Tedesco, F. S. \& Cossu, G. Stem cell therapies for muscle disorders. Curr. Opin. Neurol. 25, 597-603 (2012).
25. Azzoni, E. et al. Hemogenic endothelium generates mesoangioblasts that contribute to several mesodermal lineages in vivo. Development 141, 1821-1834 (2014).

26. Fuoco, C. et al. In vivo generation of a mature and functional artificial skeletal muscle. EMBO Mol Med 7, 411-422 (2015).

27. Godi, C. et al. Longitudinal MRI quantification of muscle degeneration in Duchenne muscular dystrophy. Ann Clin Transl Neurol 3, 607-622 (2016).

28. Astuti, P. et al. MAPK pathway activation delays G2/M progression by destabilizing Cdc25B. J. Biol. Chem. 284, 33781-33788 (2009).

29. Thornton, T. M. \& Rincon, M. Non-classical p38 map kinase functions: cell cycle checkpoints and survival. Int. J. Biol. Sci. 5, 44-51 (2009).

30. Fuoco, C. et al. Injectable polyethylene glycol-fibrinogen hydrogel adjuvant improves survival and differentiation of transplanted mesoangioblasts in acute and chronic skeletal-muscle degeneration. Skelet Muscle 2, 24-36 (2012).

31. Barreca M. M. et al. Extracellular Hsp70 Enhances Mesoangioblast Migration via an Autocrine Signaling Pathway. J Cell Physiol 2016; Epub ahead of print; https://doi.org/10.1002/jcp.25722.

32. Cassano, M. et al. Alpha sarcoglycan is required for FGF-dependent myogenic progenitor cell proliferation in vitro and in vivo. Development 138, 4523-4533 (2011).

33. Phinney, D. G. Functional heterogeneity of mesenchymal stem cells: implications for cell therapy. J. Cell. Biochem. 113, 2806-2812 (2012).

34. Lescroart, F. et al. Early lineage restriction in temporally distinct populations of Mesp1 progenitors during mammalian heart development. Nat. Cell. Biol. 16, 829-840 (2014).

35. De Angelis, L. et al. Skeletal myogenic progenitors originating from embryonic dorsal aorta coexpress endothelial and myogenic markers and contribute to postnatal muscle growth and regeneration. J. Cell. Biol. 147, 869-878 (1999).

36. Burova, E., Borodkina, A., Shatrova, A. \& Nikolsky, N. Sublethal oxidative stress induces the premature senescence of human mesenchymal stem cells derived from endometrium. Oxid Med Cell Longev 2013, 474931-474942 (2013).

37. Pomduk, K. et al. Enhanced human mesenchymal stem cell survival under oxidative stress by overexpression of secreted frizzled-related protein 2 gene. Ann. Hematol. 94, 319-327 (2015).

38. Boopathy, A. V., Pendergrass, K. D., Che, P. L., Yoon, Y. S. \& Davis, M. E. Oxidative stress-induced Notch1 signaling promotes cardiogenic gene expression in mesenchymal stem cells. Stem Cell Res Ther 4, 43-56 (2013).

39. McClowskey, T. W., Chavan, S., Lakshmi Tamma, S. M. \& Pahwa, S. Comparison of seven quantitative assays to assess lymphocytic cell death during HIV infection: measurement of induced apoptosis in anti-Fas treated Jurkat cells and spontaneous apoptosis in peripheral blood mononuclear cells from children infected with HIV. AIDS. Res. Hum. Retrovirus. 14, 1413-1422 (1998).

40. Gargioli, C., Coletta, M., De Grandis, F., Cannata, S. M. \& Cossu, G. PIGF-MMP-9expressing cells restore microcirculation and efficacy of cell therapy in aged dystrophic muscle. Nat. Med. 14, 973-978 (2008).

41. Scardigli, R. et al. Binding of sFRP-3 to EGF in thextra-cellular space affects proliferation, differentiation and morphogenetic events regulated by the two molecules. PLoS. ONE. 3, e2471-e2481 (2008). 